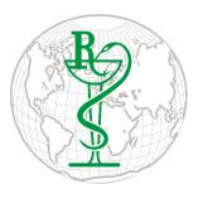

INDO GLOBAL JOURNAL OF

PHARMACEUTICAL SCIENCES

ISSN 2249- 1023

\title{
Role of Diet and Salvia miltiorrhiza to Manage Osteoporosis
}

\author{
Shelly Garg *, Luxita Sharma, Hardik Dalal \\ Dietetics and Applied Nutrition, Amity Medical School, Amity University, Gurugram, Haryana, India (122051)
}

Address for Correspondence: Shelly Garg, gargshelly8795@gmail.com

\begin{abstract}
Received:
30.01.2020

Accepted:

23.06.2020

Published:

30.03.2021

Keywords

Osteoporosis,

Women, Old age,

Salvia

miltiorrhiza,

Diet, Osteoblast,

Bones,

Menopause.

ABSTRACT: The main aim of this study is to get information about the effect of Salvia miltiorrhiza on the bone health of individuals affected from osteoporosis, and also the prevalence of osteoporosis in the different parts of the globe and also to find out its effects and the group of people affected. Different studies are being carried out to find the method of reduction of stiffness and pain in the joints. Osteoporosis is a silent disease and symptoms are not easily visible until complete development, but the major symptom is bone fracture even when a little force is exerted on the bone. It affects a total combined population of about 75 millions in Japan, Europe and USA. The combined highest prevalence of osteoporosis is of America and Europe, which is of around 51 percent of the total global population. This rapid increase in the number of cases every year makes it important for us to gain information about osteoporosis in the post-menopausal women, and trace the ways with which it can either be prevented or treated with the help of pharmacological active herb namely Salvia miltiorrhiza along with dietary modifications and changes in the lifestyle. () 2020 iGlobal Research and Publishing Foundation. All rights reserved.

Cite this article as: Garg, S.; Sharma, L.; Dalal, H. Role of Diet and Salvia miltiorrhiza to Manage Osteoporosis. Indo Global J. Pharm. Sci., 2021; 11(1): 42-46. DOI: http://doi.org/10.35652/IGJPS.2021.111006.
\end{abstract}

\section{INTRODUCTION}

A disease in which there is a marked decrease in the bone strength leading to the increased risk of bone fractures is termed as osteoporosis. The word osteoporosis is derived from two Greek words "ostoun" and "poros" meaning bones and pores respectively, and hence word osteoporosis was coined referring to a condition in which bones become porous. It is defined as bone density of 2.5 or more standard deviations less than that of a normal young adult. Bone density is measured with the help of dual-energy X-ray absorptiometry [1]. Elderly fracture is most commonly caused because of osteoporosis. There is a marked decrease in the skeletal bone mass resulting in bone deformities and severe pain. The pore size of the bone structure of an osteoporotic patient is much larger in comparison to normal [2]. Most of the times, bones of spine, forearm and hips get fractured, and even a minor strain can lead to bone fracture. It arises due to abnormally low bone mass and abnormally high bone decalcification or bone loss. Calcification and decalcification of bones takes place simultaneously, during normal conditions there is equilibrium in the process of calcification and decalcification of bones. After the age of 40 years, this equilibrium shifts to the negative side and more rapid decalcification leading to the decrease in bone mineral density and thus causing osteoporosis [3]. An important factor, which defies the risk of the disease is, Bone mineral density, and is influenced by lifestyle affecting factors like improper nutrient intake, physical inactivity or sedentary lifestyle, chronic alcoholism and smoking [4]. After the age of 30, bone mass of women decreases more rapidly than men [5]. Osteoporosis is most prevalent in women over the age of 50 years and is post menopausal, but younger women, men, pregnant women and children can also be struck with this disease at any stage of life [6]. It results in a pain for a prolonged period of time which without treatment last life long, pain results in decreased mobility. It can occur at any age, but it comes more with menopause because after menopause body estrogen levels fall down and results in decalcification of bones. Apart from age and hormone estrogen, other factors contributing in the rapid increase of the disease are sedentary lifestyle, smoking, anorexia, hyperthyroidism, kidney disorders, alcoholism, and surgeries involving removal of ovaries [7][8]. Also, use of some medications like glucocorticosteroids, selective serotonin uptake inhibitor, antiseizure medicines and 


\section{Indo Global Journal of Pharmaceutical Sciences, 2021; 11(1): $42-46$}

chemotherapy increases the risk of getting pores in the bones [6]. This disease has no major signs and symptoms, but common symptom includes fractures either symptomatic or asymptomatic [9]. Calcium intake decreases with the advancement of age, and more of calcium is excreted via urinary tract due to the absence or low levels of estrogen. So, regular medical examinations, after the age of 40 years, should be done so as to check the presence of the disease [10]. Breakage of bones or fractures is not obvious in all cases of osteoporosis, but osteoporosis increases the risk of bone breakage with even a minor force [11]. From the stats of a report given by National Osteoporosis Foundation, it was found that one out of two women over the age of 50 years are either at the risk or are suffering from osteoporosis [12]. Globally, millions of people are affected with osteoporosis making a global burden of 200 million, affecting women more than men [13].

\section{BACKGROUND}

Osteoporosis is one of the top three leading causes of deaths of women over the world. From a study conducted on healthy males over the age of 50 years, it was found that only 8.5 percent of the total healthy males were affected with osteoporosis [14]. In year 2013, 50 million people in India were found to be either having low bone density or are osteoporotic [15]. Prevalence rate of osteoporosis in Pakistan is 16 percent [16]. From the scores of T-test conducted in Sri Lanka, it was found that $44.9 \%$ women were osteoporotic [17]. From reports, 6.6- 19.3 percent population is osteoporotic. In Hong Kong, 7 percent men and 34.1-37 percent women, both over the age of 50 years are affected with osteoporosis. While 11.4 percent women and 1.6 percent men were osteoporotic in Taiwan [18]. Asian population is at a high risk of developing the disease than their Caucasian counterparts [19]. In European countries like France, Germany, Spain, Italy, UK and Sweden, around 3.8 million, 5.3 million, 2.8 million, 4 million, 3.5 million and 500,000 individuals are osteoporotic respectively. Overall, around 20 million people in Europe are osteoporotic [20]. In Europe and United states, around 30 percent post-menopausal women are suffering from the ill effects of the disease. Worldwide about 200 million women are either having osteoporosis or are at the verge of the disease. And, annually it causes more than 8.9 million fractures across the globe. According to recent statistics, one in 3 women is osteoporotic. Globally, rate of fracture due to osteoporosis is one every 3 seconds [21]. There are numerous causes of osteoporosis globally. In country like India, the reasons for high prevalence rate of osteoporosis are because of low levels of vitamin $\mathrm{D}$, around 80 percent of population has vitamin D levels below $20 \mathrm{ng} / \mathrm{mL}$. Other reason of osteoporosis in India are menopause, calcium deficiency, age, change in lifestyle, less or no physical activity, consumption of glucocorticoids, dressing habits (less exposure to sunlight leads to less absorption of vitamin D.), low bone mineral density (BMD) and bone mass and very less or no knowledge about the disease and its preventive measures [22]. In other Asian countries the major culprit behind the high prevalence rate of the disease are reduced BMI (Body Mass
Index), low bone mineral density, low levels of estrogen due to menopause, age, inadequate or less nutrient intake, lack of exercise and sedentary lifestyle, body stature, gender, bone decalcification and urbanization [20][23][24][25]. Asians and Europeans are more prone to the disease because of their ethnicity [26]. Other risk factors of the disease are heredity and genetics, with a family history of the disease, the risk of getting it also increased by 25 to 80 percent [27]. Excess alcohol consumption, vitamin D deficiency, malnutrition, smoking, high protein diet of vegetable origin (high protein diet causes excretion of more calcium in urine.), sedentary lifestyle, BMI below normal, low socio-economic status, duration of breast-feeding, exposure to heavy metals, soft drinks and proton pump inhibitors also leads to the development of the disease. Use of certain medications like steroids, glucocorticoids, anticoagulants, thiazolidinediones and aluminium containing antacids also contribute to osteoporosis. Few medical conditions like surgical removal of ovaries, premature ovarian failure, and immobilization due to fracture, Turner syndrome, anorexia nervosa, Cushing syndrome, hyperparathyroidism, hyperthyroidism, diabetes, adrenal insufficiency, malnutrition and rheumatoid arthritis favor the chances of being osteoporotic [28].

\section{DIETARY MANAGEMENT}

Due to age, reduced nutrition, reduced absorption of nutrients, proper care of diet and nutrition are to be taken care of. Special care of nutrition should be taken in childhood, and medications which promote demineralization of bones are avoided. Proper medication, exercise along with diet therapy helps in relieving pain and helps in delaying the disease [6]. Diet loaded with calcium and vitamin D either naturally or in the form of supplementation helps in reducing the severity of the disease [29]. All the nutrients should be provided keeping in mind the nutrient requirements and associated health conditions. Energy needs are provided by keeping in mind the age, BMI and nutrient absorbing power of the patient. Generally, calorie count of $1800-2000 \mathrm{Kcal}$ is given. Adequate amount of $1 \mathrm{~g} / \mathrm{kg}$ body weight of protein should be provided in every stage of life for proper bone development and maintenance of bone strength [30]. Protein should be of vegetarian source, as more of non vegetarian source of protein causes more calcium excretion in urine. Non vegetarian foods can be given but in moderation. Milk, dairy products, soy, small edible bones, eggs are given as a source of protein [31]. Carbohydrates should contribute 55-65 percent of carbohydrate. Carbohydrate should be a mixture of simple and complex carbohydrates. Dietary fiber should not be provided in large amounts as fiber forms chelates with calcium and makes calcium unavailable for absorption in the gastrointestinal tract [32]. Fortified breakfast cereals, fruits and vegetables, especially fruits and vegetables rich in phosphorous should be given. Fats are to be given in moderation not more than 20 percent or 60 grams. This is because with more fat in diet adiposity increases and bone fragility also increases and the risk of fracture and bone deformities increases [33]. More amount of PUFA (Poly Unsaturated Fatty Acid) should be given, as PUFA increases 


\section{Indo Global Journal of Pharmaceutical Sciences, 2021; 11(1): $42-46$}

the bone mineral density and reduces the risk of bone fragility [34]. Calcium is very necessary for proper bone strength and should be provided on daily basis in diet and as supplements. This is done to maintain positive calcium balance in the body. Daily calcium intake of $1200 \mathrm{mg} /$ day for age 50 and above is recommended. Vitamin D is helpful in absorbing calcium by the body and bones, so is also required. Per day requirement of Vitamin D is 400 IU. Sunlight is the best source of vitamin D, apart from that fortified foods and supplements are given [35]. Phosphorous intake is to be maintained, as increased phosphorous reduces urinary calcium loss and promotes fecal calcium loss [36]. The RDA (Recommended Dietary Allowances) for phosphorous is $700 \mathrm{mg} /$ day for healthy adults and serum phosphorous levels should be between the range of 2.5 to $4.5 \mathrm{mg} / \mathrm{dL}$ [37]. Foods like milk, pulses, fruits, vegetables, egg, fish, nuts and seeds are included in the diet. Bread, pasta, cereals, potato, dairy products are also given [38]. Foods fortified with calcium and vitamin D, like fortified juice, fortified dairy products, yogurt, cream, cheese is given. Foods like common salt are avoided, as more of salt leads to calcium loss over a period of time. Some herbs and spices are given like black pepper, cardamom, ginger, turmeric and cumin seeds for their health benefits [39]. Processed, canned and baked foods are avoided. Carbonated beverages, caffeinated drinks are avoided. Tea is better tolerated than coffee, as caffeine decreases the body's ability of calcium retention [4]. For the management of osteoporosis in postmenopausal women, 'The Indian Menopausal Society' put forward some guidelines according to the time period (in years) after menopause. Only primary care and preventive measures like proper nutrition, supplementation of calcium and vitamin $\mathrm{D}$ and exercise are done in case of less than 5 years of menopause. While, in case of menopause more than 5 years, requires proper medical care, food and nutrient supplementation [12]. Along with nutrient therapy, medications are also necessary. Medicines can be in the form of herbs.

\section{SALVIA MILTIORRHIZA}

Salvia miltiorrhiza is a perennial plant belonging to the genus Salvia, and is a Chinese herb used extensively for its medicinal properties. Some common names of the herb are Chinese sage, red sage, danshen or tan shen [40]. Length of plant reaches up to $1,200 \mathrm{~m}$, and belongs natively to China and Japan. Tanshinone IIA, a constituent, is present in abundance in the herb. Other, chemical constituents include tanshinone I, salvianolic acid and dihydrotanshinone [41]. Flowers are light purple to lavender blue in color and are grown in whorls. Herb grows well in less sunlight and well-draining land [42]. In traditional Chinese medicine, Red sage in combination with other herbs is used to treat skeletal disorders. With advancement in technology, a bone targeting active compound has been isolated from the sage roots. So, is used in the treatment of osteoporosis. The anti-osteoporotic effect is seen from its action on liver and blood stasis, which is its main therapeutic effect on osteoporosis. Many therapeutic trials have been done and it was found that it helps in the treatment of post-menopausal and secondary osteoporosis [43]. From a study conducted on rats, it was found that red sage is a natural product for the treatment of osteoporosis. This effect is because of its anti-oxidative stress effect by regulating osteoclast number and maturation [44]. From another study done on ovariectomized rats, results obtained suggest strong preventive effect of the sage herb in bone loss induced by removal of ovaries in rats [45]. From another study, it was found that tanshinone, a compound found in the red sage extract has good antiosteoporotic activity [46]. From a research on the "effects of medicinal herb Salvia miltiorrhiza on osteoblastic cells in vitro", it was found that the herb has shown a marked increase in the gene expression by increasing alkaline phosphatase and osteocalcin activity and thus promoting bone formation, and also decreasing the bone resorption activity [47]. From another study conducted on cultivated human osteoblasts, it was concluded that the herb has bone Gla protein production, by osteoblast, promoting activity. It also helps in increasing the calcium ion concentration in the osteoblastic cells. And, by doing so, the herb aids in osteoblast maturity along with bone formation and mineralization [48]. From experiments, researchers observed that red sage extract combined with calcium supplement in the liquid form helps in reducing bone loss and promoting bone formation and elevated bone mineral density [49]. Elevation of bone mineral density by 35 percent has been seen with the consumption of red sage extract by the Canadian researchers [50]. Compound which serves as a best osteoporotic activity is Tanshinone IIA sulfonic sodium (To6). From results published in the Journal of Bone and Mineral Research, it was concluded that To6 helped in increasing the number of osteoblast cells by 28 percent and also an increase in the bone mineral density by 35 percent, without showing any side effects [51].

\section{CONCLUSION}

Osteoporosis is the porous bone disease and is found in old age especially in postmenopausal women. It is caused due to reduced in bone mineral density because of increased demineralization of bones, and leads to easy fractures even when little pressure is exerted on the bones. Other contributing factors are low levels of estrogen, alcohol, obesity, smoking, certain medications and surgeries. It is identified with dual energy X-ray absorptiometry. From the above review, the prevalence of osteoporosis has been found, which is about 200 million women suffering globally. About one in three women are osteoporotic. It can be managed with proper medication, exercise and dietary management. Diet rich in calcium and vitamin D is given along with supplementation. All nutrients are given in good amounts, so as to avoid deficiencies in old age. Normal calorie diet of $1800-2000$ Kcal is given because of obesity and decreased absorption capacity. Mixture of complex and simple carbohydrates is given along with high biological value protein, PUFA (Poly Unsaturated Fatty Acid), MUFA (Monounsaturated Fatty Acid), vitamins and minerals. Proper care is taken of sodium and phosphorous, as when increased, increases the rate of decalcification and excretion of calcium in urine. Apart from dietary medications and chemical enriched medicines, herb Salvia miltiorrhiza has been found of great importance in curing osteoporosis by reducing 


\section{Indo Global Journal of Pharmaceutical Sciences, 2021; 11(1): 42-46}

decalcification of bones and improving bone mineral density by 35 percent, without any side-effects. Salvia miltiorrhiza is commonly called as red sage. Compounds found in the herb are tanshinone IIA, tanshinone I, salvianolic acid and dihydrotanshinone. Best osteoporotic activity is shown by tanshinone IIA. Taking red sage capsules or extracts along with calcium (particularly in liquid form) helps in the prevention of osteoporosis in pre-osteoporotic individuals. It also helps in reducing the severity of the disease by increasing calcification of bones and reducing porosity.

\section{ACKNOWLEDGEMENTS}

None

\section{FUNDING}

Authors have not declared any external funding source here.

\section{CONFLICT OF INTEREST}

The authors declare that they have no conflict of interest.

\section{DATA AVAILABILITY}

Not declared.

\section{REFERENCES}

[1] WHO Scientific Group on the Prevention and Management of Osteoporosis (2000: Geneva, Switzerland) (PDF). 2003; 31: 7. ISBN 978-9241209212.

[2] Bharadwaj, Sriram. Mapping of Indian Research Output on Osteoporosis. Journal of Midlife Health, 2013; 276-283.

[3] Simonelli, C et al. ICSI Health Care Guideline: Diagnosis and Treatment of Osteoporosis, 5th edition, Institute for Clinical Systems Improvement, 2006; 37: 32-35.

[4] Sharma, S., Khandelwal S. Effective Risk Assessment Tools for Osteoporosis in the Indian Menopausal Female. Journal of Midlife Health, 2010; 1: 79-85.

[5] "6.6 Exercise, Nutrition, Hormones, and Bone Tissue". Anatomy \& Physiology. Openstax CNX. 2013. ISBN 978-1-938168-13-0.

[6] Jones, D., Roach, P., Hartmann, J., Setter, J. English Pronouncing Dictionary, Cambridge: Cambridge University Press, ISBN 978-3-12-539683-8.

[7] Sinnesael, M., Claessens, F., Boonen, S., Vanderschueren, D. Novel Insights in the Regulation and Mechanism of Androgen Action on Bone. Current Opinion in Endocrinology, Diabetes and Obesity, 2013; 20(3): 240-44.

[8] Sinnesael, M., Boonen, S., Claessens, F., Gielen, E., Vanderschueren, D. Testosterone and the Male Skeleton: a Dual Mode of Action. Journal of Osteoporosis, 2011:1-7.

[9] Old, J.L., Calvert, M. Vertebral Compression Fractures in the Elderly. American Family Physician, 2004; 69(1): 11116 .

[10] Sellmeyer, D.E., Stone, K.L., Sebastian, A., Cummings, S.R. A High Ratio of Dietary Animal to Vegetable Protein Increases the Rate of Bone Loss and the Risk of Fracture in Postmenopausal Women. Study of Osteoporotic Fractures Research Group. American Journal of Clinical Nutrition, 2001; 73(1):118-22.
[11] Agarwal, N., Raveendran, A. et al. Prevalence and Related Risk Factors of Osteoporosis in Pre and Postmenopausal Indian Women. Journal of Midlife Health, 2011; 2(2): 8185.

[12] Patni, R. Normal BMD Values for Indian Females Aged 20- 80 yrs. Journal of Midlife Health, 2010; 1: 70-3.

[13] International Osteoporosis Foundation. Accessed from https://www.iofbonehealth.org/ on 5 February 2019.

[14] Das, S., Sharma, L., Ajmera, P. Prevalence and management of osteoporosis in menopausal women through dietary modifications. International Journal of Home Science, 2017; 3(1): 355-358.

[15] Mithal, A., Kaur, P. Osteoporosis in Asia: A Call to Action. Current Osteoporosis Report. 2012; 10: 245-7.

[16] Sultan, A., Khan, D.A., Mushtaq, M., Hassan, M.U. Frequency of Osteoporosis and its Associated Risk Factors in Postmenopausal Women in Clinical Practice at Rawalpindi. Pakistan Journal of Pathology, 2006; 17: 115118.

[17] Lekamwasam, S., Wijayaratne, L., Rodrigo, M., Hewage, U. Prevalence of Osteoporosis Among Postmenopausal Women in Sri Lanka; a Cross-sectional Study. International Journal of Rheumatic Diseases, 2007; 10(3): 234-38.

[18] Wang, Y., Tao, Y., Hyman, M.E., Li, J., Chen, Y. Osteoporosis in China. Osteoporosis International, 2009; 20(10): 1651-1662.

[19] The Asian Audit Epidemiology, Costs and Burden of Osteoporosis in Asia 2009. Accessed from https://www.iofbonehealth.org/sites/default/files/PDFs/Aud it\%20Asia/Asian_regional_audit_2009.pdf 09 February 2019.

[20] Data and Publications by International Osteoporosis Foundation. Accessed from https://www.iofbonehealth.org/facts-statistics on 09 February 2019.

[21] Melton III, L.J., Chrischilles, E.A., Cooper, C., Lane, A.W., Riggs, B.L. Perspective: How Many Women have Osteoporosis? Journal of Bone and Mineral Research, 1992; 7: 1005-10.

[22] Mithal, A., Bansal, B., Kyer, C.S., Ebeling, P. The AsiaPacific Regional Audit- Epidemiology Costs, and Burden of Osteoporosis in India 2013: a Report of International Osteoporosis Foundation. Indian Journal of Endocrinology and Metabolism, 2014; 18(4): 449-454.

[23] Ejaz, S., Mahmood, A., Qureshi, A.A., Ali, M. Prevalence of Osteoporosis and Osteopenia Among Pakistani Pre and Post Menopausal Women. Journal of Dental and Medical Sciences, 2012; 2(6): 12-17.

[24] Siribaddana, S.H., Lekamwasam, S. Osteoporosis in Sri Lanka. Clinical Calcium, 2004; 14(9): 128-33.

[25] Junhong, P. et al. Chinese Population, the Diagnostic Criteria of Primary Osteoporosis and the Incidence of Osteoporosis in China. Chinese Journal of Osteoporosis, 2002-01.

[26] Melton, L.J. Epidemiology Worldwide. Endocrinology and Metabolism Clinics of North America, 2003; 32 (1): 1-13.

[27] Raisz, L. Pathogenesis of Osteoporosis: Concepts, Conflicts, and Prospects. Journal of Clinical Investigation, 2005; 115(12): 331825.

[28] Thulkar, J., Singh, S. Preventable Risk Factors for Osteoporosis in Post-Menopausal Women. Journal of Midlife Health, 2016; 7: 108-113.

[29] "Drugs for Postmenopausal Osteoporosis". The Medical Letter on Drugs and Therapeutics, 2014; 56(1452): 91-96. 


\section{Indo Global Journal of Pharmaceutical Sciences, 2021; 11(1): $42-46$}

[30] Rizzoli, R., Bonjour, J.P. Dietary Protein and Bone Health. Journal of Bone Mineral Research, 2004; 19: 527-31.

[31] Agarwal, N., et al. Prevalence and Related Risk Factors of Osteoporosis in Pre and Postmenopausal Indian Women. Journal of Midlife Health, 2011; 2(2): 81-85.

[32] Reinhold, J.G., Faradji B, Abadi P, Ismail-Beigi F. Decreased Absorption of Calcium, Magnesium, Zinc and Phosphorous by Humans due to Increased Fiber and Phosphorous Consumption as Wheat Bread. Journal of Nutrition, 1976; 106: 493-503.

[33] Kawai, M, de Paula, F.J.A., Rosen, C.J. New Insights into Osteoporosis: Bone Fat Connection. Journal of Internal Medicine, 2012; 272(4): 317-329.

[34] Longo, A.B., Ward, W.E. PUFA's, Bone Mineral Density and Fragility Fracture: Findings from Human Studies. Advances in Nutrition, 2016; 7(2): 299-312.

[35] Sunvecz, J.A. The Use of Calcium and Vitamin D in the Management of Osteoporosis. Therapeutics and Clinical Research Management, 2008; 4(4): 827-836.

[36] Diet and Health: Implications for Reducing Chronic Disease Risk. National Academy Press, Washington, D.C. 1989. Chapter 23 Osteoporosis. Accessed on 30 January 2019.

[37] Linus Pauling Institute: Micronutrient Information Center. Accessed from https://lpi.oregonstate.edu/mic/minerals/phosphorus on 2 February 2019.

[38] Sharma, L., Ajmera, P. Organoleptic and Physicochemical Properties of Tarts Developed from Quinoa, Chickpea and Oats Flour and Their Ranking by Topsis Method. Curr Res Nutr Food Sci, 2019; 7(2).

[39] Sharma, L., Garg, V., Ajmera, P., Maria. Protective role of Piper nigrum in health and healing of diseases. International Journal of Food Science and Nutrition, 2018; 3(2): $84-88$

[40] Ji, X.Y., et al. Effects of Salvia miltiorrhiza After Acute Myocardial Infarction in Rats. Novel Compounds from Natural Products in the New Millennium: Potential and Challenges. Singapore: World Scientific, 2004: 183-95.

[41] Danshen. Accessed from https://www.drugs.com/npp/danshen.html $\quad$ on 22 January 2019.

[42] Lee, W.Y., et al. Cytotoxic Effects of Tanshinones from Salvia miltiorrhiza on Doxorubicin-Resistant Human Liver Cancer Cells. Journal of Natural Products, 2010; 73(5): 854-9.

[43] Yubo, G., et al. Salvia miltiorrhiza: an Ancient Chinese Herbal Medicine as a Source for Anti-Osteoporotic Drugs. Journal of Ethnopharmacology, 2014; 155(3): 1401-1416.

[44] Cui, Y., et al. Characterization of Salvia miltiorrhiza Ethanol Extract as an Anti-Osteoporotic Agent. International Society for Complementary Medicine Research, 2011: 120.
[45] Chae, H.J., et al. Prevention of Bone Loss in Ovariectomized Rats: the Effect of Salvia miltiorrhiza Extracts. Journal of Immunopharmacology and Immunotoxicology, 2004; 26(1): 134-44.

[46] Kim, H.K., et al. The Correlation of Salvia miltiorrhiza Extract- Induced Regulation of Osteoclastogenesis with the Amount of Components Tanshinone I, Tanshinone IIA, Cryptotanshinone, and Dihydrotanshinone. Journal of Immunopharmacology and Immunotoxicology, 2008; 30(2): 347-64.

[47] Chin, A., Yang, Y., Chai, L., Wong, R.W.K, Rabie, A.B.M. Effects of Medicinal Herb Salvia miltiorrhiza on Osteoblastic Cells in Vitro. Journal of Orthopaedic Research, 2011; 29(7): 1059-63.

[48] Effects of Salvia miltiorrhiza on Production of Osteocalcin and Concentration of Calcium ion in Human Osteoblasts. Accessed from http://en.cnki.com.cn/Article_en/CJFDTOTALSJKX201302025.htm on 28 January 2019.

[49] Taking Liquid Calcium with Red Sage can Build Stronger Bones. Accessed from https://phytonutrients.news/201809-18-taking-liquid-calcium-with-red-sage-can-buildstronger-bones.html on 5 February 2019.

[50] Bone Cure: this Chinese Herb may Treat People with Osteoporosis. Accessed from https://www.hindustantimes.com/health/bone-cure-thischinese-herb-may-treat-people-with-osteoporosis/storymDXX6dNJIRE9cw6mhXRqMJ.html on 5 February 2019.

[51] Red sage: Could this Ancient Chinese Herb Prevent Bone Loss. Accessed from https://www.pharmaceuticaltechnology.com/features/red-sage-ancient-chinese-herbprevent-bone-loss/ on 7 February 2019. 